\title{
Establecimiento de banco de forraje hidropónico como suplemento alimenticio para ganado bovino del Casanare en época de sequía
}

\section{Establishment of a hydroponic green forage bank as feed supplement for bovine cattle of Casanare in drought age}

Diana Marcela Africano Guevara
Ingeniero Agrícola, Fundación universitaria de San Gil. Yopal -Casanare-Colombia.
(Autor para correspondencia)
dianaafricano@unisangil.edu.co
Andrés Francisco Garcia Orozco
Ingeniero Agrícola, Fundación universitaria de San Gil. Yopal-Casanare-Colombia
andresgarciao@unisangil.edu.co
Wilmer Leonardo Velazques Vargas
Ingeniero Agrícola, Docente investigador, Facultad de Ciencias Naturales.
Fundación universitaria de San Gil. Yopal -Casanare-Colombia
wvelasquez@unisangil.edu.co

\section{Resumen}

La ganadería bovina en Casanare ha sido afectada por condiciones climáticas adversas como sequias prolongadas e inundaciones que han causado; baja disponibilidad de alimento, escasez nutricional de forrajes y pastos en las fincas. Por tanto, en esta investigación se estableció una estrategia para mitigar la falta de alimento por medio de banco de forraje hidropónico con el fin de optimizar y estabilizar la producción de alimento para ganadería bovina en Casanare. De esta manera el objetivo de este estudio fue evaluar y comparar por medio de prueba T student una especie vegetal Zea Maíz variedad Guacavia en dos tipos de fertilizantes; "fertilización química T1 y fertilización orgánica T2" , se analizó; altura, ganancia de biomasa fresca y eficiencia de conversión, para ello se realizó mediciones diarias en altura donde se tomó 5 plántulas por bandeja y en biomasa el peso diario al tapete de forraje, a su vez para conocer el valor nutricional se envió 500 gr de muestra de cada tratamiento para su posterior análisis bromatológico. En los dos tratamientos no tuvo efecto significativo ( $\mathrm{p}>0.05)$ sobre las variables, por otro lado, se sembró $2 \mathrm{~kg}$ de semilla por bandeja y al día once se obtuvo en el tratamiento T2 $9.1 \mathrm{~kg}$ de biomasa, $38.7 \mathrm{~cm}$ de altura y $11.5 \%$ de proteína a base seca, mientras que en el tratamiento T1 logro mejores resultados, $11.2 \mathrm{~kg}$ de biomasa, $45 \mathrm{~cm}$ de altura y $19.1 \%$ de proteína a base seca.

Palabras clave: altura; forraje hidropónico; ganadería; suplemento 


\begin{abstract}
Cattle ranching in Casanare has been affected by adverse climatic conditions such as prolonged droughts and floods that have caused; low availability of food, nutritional shortage of forages and pastures on farms. Therefore, in this research a strategy was established to mitigate the lack of food by means of a hydroponic forage bank in order to optimize and stabilize the production of cattle feed in Casanare. In this way, the objective of this study was to evaluate and compare, through the T student test, a Zea Maiz variety of Guacavia plant in two types of fertilizers; "Chemical fertilization T1 and organic fertilization T2", was analyzed; Height, gain of fresh biomass and conversion efficiency, for this daily measurements were made in height where 5 seedlings per tray were taken and in biomass the daily weight to the forage mat, in turn to know the nutritional value was sent 500 gr of sample of each treatment for its later bromatological analysis. In both treatments there was no significant effect $\left(\mathrm{p}_{i}, 0.05\right)$ on the variables, on the other hand, $2 \mathrm{~kg}$ of seed was planted per tray and on the eleventh day it was obtained in the treatment $\mathrm{T} 29.1 \mathrm{~kg}$ of biomass, $38.7 \mathrm{~cm}$ of height and $11.5 \%$ of protein based dry, while in treatment T 1 achieved better results, $11.2 \mathrm{~kg}$ of biomass, $45 \mathrm{~cm}$ in height and $19.1 \%$ of protein based dry.
\end{abstract}

Keywords: height; hydroponic forage; cattle raising; supplement

\section{Introducción}

La actividad ganadera del departamento de Casanare cuenta con una extensión en pastos y forrajes de 2,465,302 ha y un total de 1,919,200 cabezas de ganado bovino distribuidos en 14,295 predios (ICA, 2016), los cuales son afectados por el déficit de precipitación en verano y no cuenta con bancos de forraje aprovechables para alimentación de bovinos. En este sentido la alimentación ganadera tradicional la constituyen pastos nativos y forrajes, sin embargo, la disponibilidad en fincas casanareñas es baja, causado por condiciones climáticas adversas como las sequias que conlleva a escases de alimento, insuficiente calidad nutricional y aumento de precio en productos como ensilaje $\mathrm{u}$ henolaje que se encuentra en el mercado, afectando así la rentabilidad del ganadero.

El forraje verde hidropónico FVH, representa según Vargas (2008) una alternativa eficaz, económica y segura en la nutrición animal ya que es un suplemento de alta palatabilidad, digestibilidad y brinda excelente contenido nutricional de proteína, energía, vitaminas y minerales según requerimiento animal (Ramírez, et al., 2017), esta técnica consiste en la producción de biomasa vegetal obtenida del crecimiento inicial de plantas por un lapso de tiempo de 9 a 15 días después de la siembra (Müller, et al., 2006) a partir de semillas aptas, especialmente leguminosas y cereales como es el caso de la Hordeum Vulgare, Triticum, Avena Sativa, Oryza Sativa y Zea Mays (FAO, 2001), a su vez, Ceballos y García (1992) afirma que se tiene en cuenta para seleccionar la semilla; la adaptabilidad climática y la necesidad del productor. La especie vegetal más usada para producir FVH es el maíz, por sus altos contenidos de carbohidratos solubles, alta digestibilidad y aceptabilidad por el animal, esta especie produce a cerca de 30,000 kg de forraje verde por hectárea, con alto valor nutricional en materia seca (23-36\%), contenido de proteína cruda aceptable (6-10\%), fibra calculada por el método de detergente neutro entre 57 y $72 \%$ a una edad de corte de 75 días (Guzmán, et al., 2003), por tanto, al suplementar durante cinco meses en época de verano a 10 novillos de $380 \mathrm{~kg}$ de peso se suministra $30 \mathrm{~kg}$ diario por animal.

Una ventaja destacable es obtener FVH durante todo el año en superficies pequeñas utilizando anaqueles y bandejas donde se siembra después del proceso de inhibición en la semilla, junto con un sustrato que proporciona soporte en el crecimiento de las raíces y conlleva a constituir una base adecuada a las plantas, los cuales pueden ser: fibra de coco, cascarilla de arroz o aserrín (Baixauli, et al., 2002), de igual forma es indispensable el aporte de macro y micro nutrientes requeridos en el crecimiento de las plantas por medio del suministro 
de fertilizante diluido mediante riego, para obtener un tapete forrajero aproximadamente en 25 días con una altura promedio de $31.14 \mathrm{~cm} \mathrm{y} 10 \mathrm{~kg} \mathrm{~m}^{2}$ de rendimiento de forraje cosechado (Pasuy, et al., 1989). De esta manera la fertilización puede ser química u orgánica siendo un factor que influye en el comportamiento de los germinados, el objetivo del estudio fue evaluar y comparar biomasa, alometria y calidad nutricional del forraje hidropónico de maíz variedad Guacavia usando dos tipos de fertilización: Biofertilizante líquido microbiano $\mathrm{y}$ fertilizante comercial para hidroponía llamado solución A-B.

\section{Materiales y métodos}

El experimento de desarrollo en época de estiaje en el departamento del Casanare del 12 al 25 de diciembre del 2017, en la hacienda la pradera, ubicada en la vereda Mata Larga del municipio de Pore del departamento de Casanare, latitud norte $05^{\circ} 65^{\prime}$ y longitud Oeste $72^{\circ} 03^{\prime}$. Se empleó un diseño experimental al azar, para el cual se realizó cinco repeticiones por cada tratamiento de fertilización; T1, fertilización química y T2, fertilización orgánica.

Para el establecimiento de FVH se construyó dos anaqueles verticales en perfil de hierro, ensamblado en tornillo y tuerca de seguridad, cada anaquel ocupa un área de $76 \mathrm{~m}^{2}$ (2 m alto x $1.26 \mathrm{~m}$ largo x $6.1 \mathrm{~cm}$ ancho) de cinco pisos con capacidad de almacenamiento para 10 bandejas, tiene 7.5 grados de inclinación en los apoyos horizontales donde son situadas las bandejas de polietileno $(59 \mathrm{~cm} \times 79 \mathrm{~cm}$ x $1.5 \mathrm{~cm}$ de profundidad). En la instalación del sistema de riego para un anaquel se utilizó; $11 \mathrm{~m}$ de tubería de PVC para todo el sistema de riego, 3 registro de bola, 7 tees, 4 codos $90^{\circ}$, una bomba de agua y 10 micro aspersores de $360^{\circ}$ de radio que se ubicaron a una altura de $32 \mathrm{~cm}$ de las bandejas hidropónicas.

En cada bandeja hidropónica de $44.6 \mathrm{~m}^{2}$ se registró durante 11 días por lectura directa altura, biomasa y eficiencia de conversión. La primera variable, se seleccionó cinco (5) plantas al azar del centro de la bandeja a fin de descartar efectos de borde para medir longitud desde la raíz adventicia hasta el ápice de la hoja. En la segunda variable se tomó el peso fresco diario del tapete en kilogramo por cada bandeja utilizando una balanza electrónica (Pesatronik) y la tercera variable se obtuvo a partir de biomasa fresca entre el peso de la semilla utilizada por bandeja (kg $\mathrm{kg}^{-1}$ ). A fin de comparar el contenido nutricional del forraje "calorías, carbohidratos, cenizas, fibra cruda, grasa cruda, humedad, proteína cruda y solidos totales" se envió una muestra de 500 gr por cada tratamiento. Los resultados obtenidos de las variables estudiadas fueron sometidos a verificación de normalidad y al cumplirse dichos supuestos, se procede a realizar un análisis de Prueba T de Student para muestras independientes $(\mathrm{p}<0.05)$, para ello se usó el programa IBM SPSS 20.

En la investigación se usaron 5 bandejas en un anaquel por cada tratamiento de fertilización, se sembró $2 \mathrm{~kg}$ de semilla germinada y 200 gr de cascarilla de arroz previamente desinfectada y distribuida uniformemente a cada bandeja, en la Tabla 1. se preparó la dosificación para los dos tratamientos en 250 litros de agua, T1 con $250 \mathrm{gr}$ del paquete A y 250 gr del paquete $\mathrm{B}$, el tratamiento T2 se aplicó $13 \mathrm{ml}$, igualmente en la Tabla 2 . se observa la concentración de nutrientes que contienen los tratamientos utilizados. Se estableció frecuencias de 4 riegos diarios a distintas horas del día por 3 minutos, desde el día de la siembra hasta el día 5 , mientras que el día 6 al día 11 los riegos fueron de 4 minutos, sin embargo, al décimo día se suspende la aplicación de fertilizante y se realiza el riego con agua para eliminar todo rastro de sales minerales sobre las hojas y raíces para que este pueda ser consumido por la reces. 
Tabla 1. Fertilizantes empleados

\begin{tabular}{cll}
\hline Nombre de tratamiento & Fertilizante empleado & Disolución realizada \\
\hline T1 & Solución nutritiva AB & $\begin{array}{l}250 \text { gr de solución B y } \\
250 \text { gr de solución A }\end{array}$ \\
\hline T2 & $\begin{array}{l}\text { Biofertilizante liquido } \\
\text { microbiano }\end{array}$ & \\
\hline
\end{tabular}

La especie usada fue maíz amarillo Guacavia (Zea Mays) sometida a cuatro (4) procesos: el primero es un análisis preliminar con el propósito de eliminar de forma manual semilla con presencia de colores extraños y presencia de olores, posteriormente el segundo paso consistió en una eliminación de semilla por flotación en agua, donde se retiró las impurezas y semillas partidas a través de un colador. Previo a la siembra, se pesó $20 \mathrm{~kg}$ de semilla para los dos tratamientos, como tercer procedimiento, se desinfecto la semilla pesada y la cascarilla de arroz mediante inmersión en solución de 50 gr de cal por litro de agua durante dos (2) minutos y enjuague con agua limpia para evitar incidencia de enfermedades, seguidamente se da inicio al último proceso que influye de manera notoria en el éxito de producción el cual es, la pre germinación con una duración total de 72 horas divididas en dos etapas.
La primera es remojo, esta consistió en humedecer la semilla en agua por 24 horas, transcurrido el tiempo de remojo se dio inicio la etapa de oreado (48 horas), para ello, utilizó un saco de yute para escurrir, airear y almacenar en una caneca con tapa ya que se sumerge la semilla en agua cada tres (3) horas por dos minutos con el objetivo de romper el estado de latencia de la semilla e inducir a una rápida germinación para asegurar un crecimiento vigoroso del forraje. Finalizado los cuatro procedimientos, se observó que el 95\% de las semillas dentro de los sacos de yute presento un promedio de $2.5 \mathrm{~cm}$ de raíz y formación del coleptile, superando el porcentaje de germinación no inferior al $75 \%$ para evitar pérdidas en los rendimientos de FVH según FAO (2001).

Tabla 2. Composición química de los fertilizantes aplicados para la producción de forraje hidropónico de maíz.

\begin{tabular}{ccccccccccccccc}
\hline Fertilización & & $\mathbf{N}$ & $\mathbf{P}$ & $\mathbf{K}$ & $\mathbf{C a}$ & $\mathbf{M g}$ & $\mathbf{S}$ & $\mathbf{F e}$ & $\mathbf{M n}$ & $\mathbf{B}$ & $\mathbf{C u}$ & $\mathbf{Z n}$ & $\mathbf{C l}$ & $\mathbf{S i}$ \\
\hline Química ppm & $\mathbf{T 1}$ & 208 & 65 & 242 & 212 & 40 & 1.4 & 3 & 0.13 & 0.1 & 0.1 & 0.11 & 0.3 & \\
\hline Orgánica \% & T2 & 1.0 & 1.1 & 0.9 & 1.1 & 1.71 & 0.13 & 1 & 0.66 & 0.1 & 0.01 & 0.02 & 0 & 65.6 \\
& & 1.2 & & & & & & & & & & & & \\
\hline
\end{tabular}

\section{Resultados y discusión}

$\mathrm{Al}$ evaluar el efecto de dos tratamientos en la producción de FVH de maíz, en relación a biomasa se obtuvo que el tratamiento T1 no genero diferencia estadísticamente significativa $(\mathrm{p}<0.05)$ al tratamiento $\mathrm{T} 2$, no obstante, el peso promedio de los tratamientos fue de: $11.2 \mathrm{~kg}$ de biomasa fresca para el T1, mientras que el tratamiento $\mathrm{T} 2$ obtuvo un valor promedio inferior de $9.2 \mathrm{~kg}$ forraje verde (Gráfica 1), de acuerdo con Hernández (2016) se produce entre 10 y 14 kilogramos de forraje en un lapso de 8 a 10 días con $2 \mathrm{~kg}$ de semilla de maíz, por tanto, el tratamiento T1 contenían suficiente concentración de nitrógeno, ya que la solución nutritiva utilizada presenta una excelente concentración mineral (Tabla 2) debido a que sobrepasa el rango de nitrógeno reportado por FAO (2001) de 200 ppm. Sin embargo, los niveles de crecimiento y producción de biomasa en plantas fertilizadas con té de compost y de vermicompost se deben principalmente a que los nutrientes contenidos en estas soluciones se encuentran en forma iónica y por lo tanto están disponibles para las plantas (Pant, et al., 2009); (Theunissen, et al., 2010). 


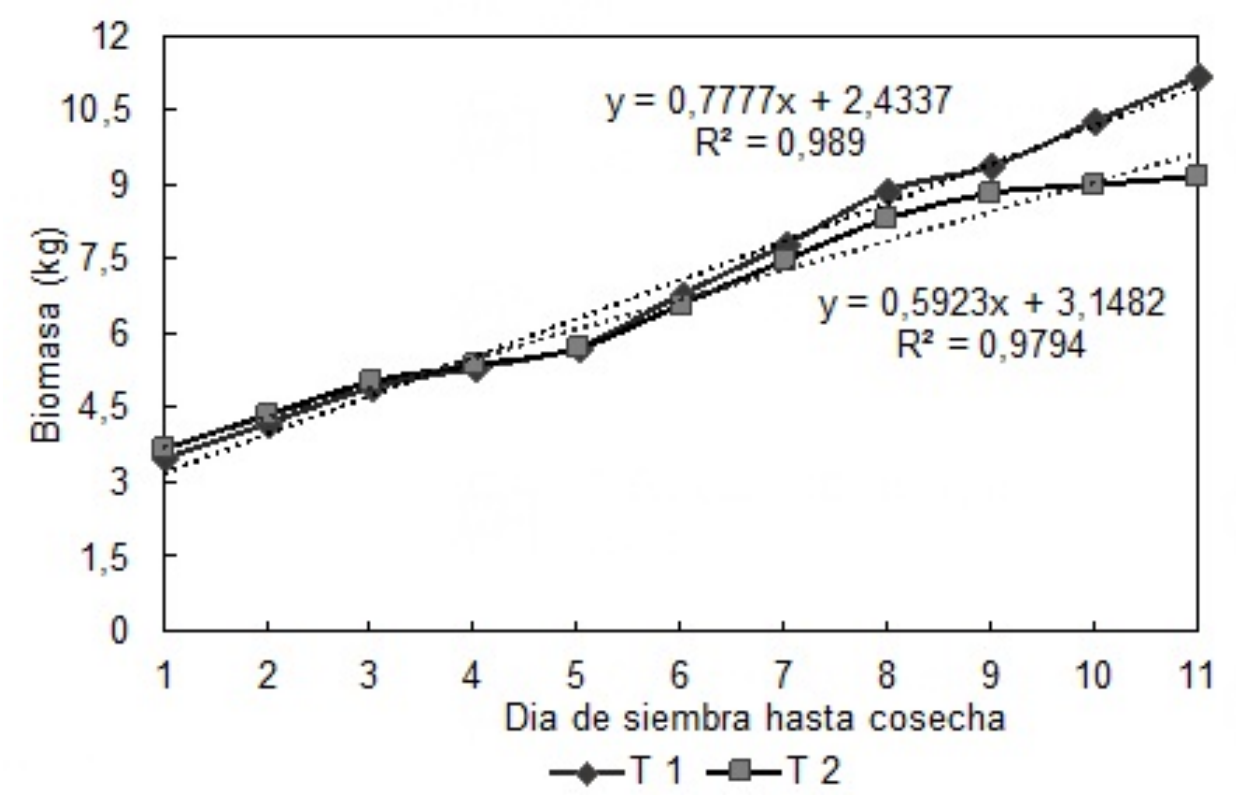

Gráfica 1. Comparación de biomasa en dos tratamientos

La eficiencia de conversión $\left(\mathrm{kg} \mathrm{kg}^{-1}\right)$ alcanzado a lo obtenido. Otros autores reiteran que la eficiencia los 11 días de cosecha y semilla sembrada no presento depende de la calidad de germinación de la semilla diferencia significativa $(\mathrm{p}<0.05)$, en la figura 2. y la variedad de maíz (blanco o amarillo), pues en se destacó el tratamiento $\mathrm{T} 1$ con $5.6 \mathrm{~kg}$ sobre el un rango de $5 \mathrm{~kg} \mathrm{~kg}^{-1}$ es clasificado como nivel de tratamiento T2 $(4.6 \mathrm{~kg})$, sin embargo, se encontró buen rendimiento, pero considera de 6 a $7 \mathrm{~kg} \mathrm{~kg}^{-1}$ que el máximo resultado de rendimiento de forraje un nivel óptimo según (Ramírez, et al., 2017), de es de $3.86 \mathrm{~kg}$ por cada kilogramo de semilla (Rivera, esta manera se deduce que el tratamiento T1 fue de et al., 2010), lo que traduce a una eficiencia inferior a buen rendimiento.

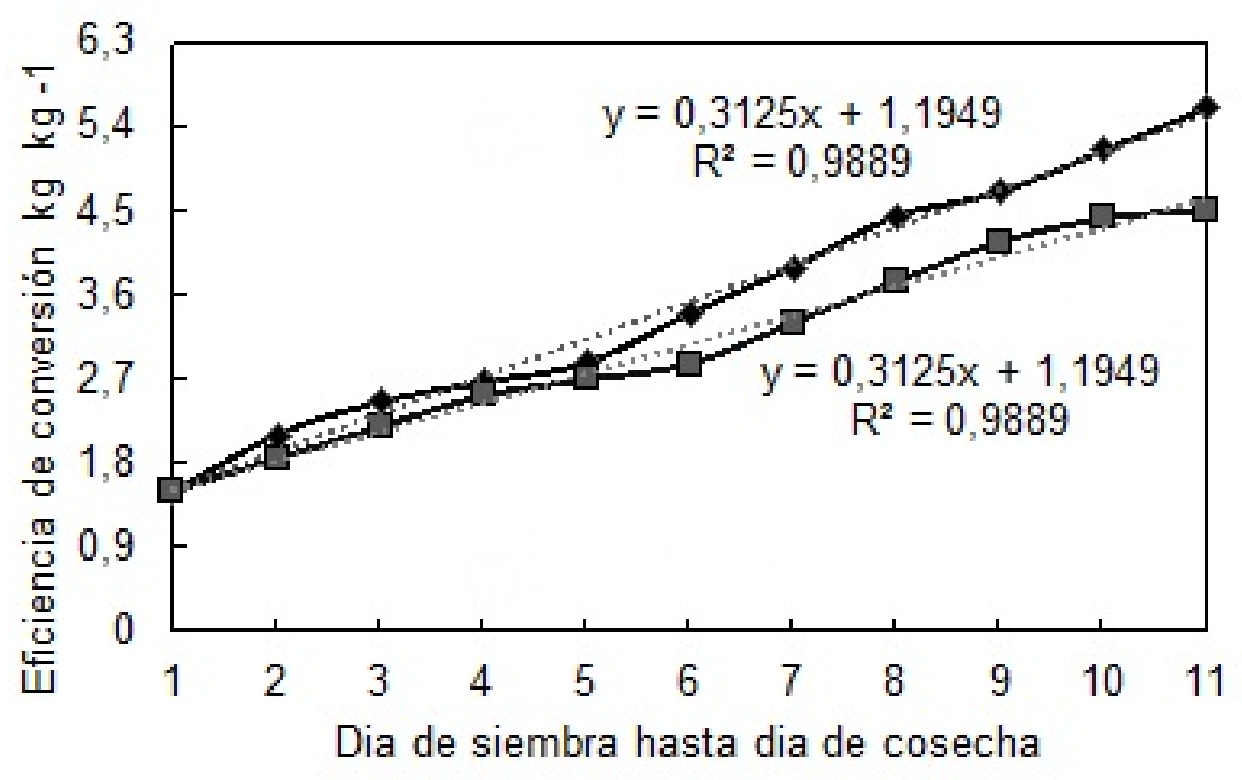

\section{$\rightarrow-\top 1-\square-T 2$}

Gráfica 2. Eficiencia de conversión diaria de forraje hidropónico en dos tratamientos 
Respecto a la variable altura no se observó diferencia significativa para los dos tratamientos, pero el tratamiento $\mathrm{T} 1$ y $\mathrm{T} 2$ en los primeros siete días obtuvieron resultados promedios similares, sin embargo, se obtuvo mayor crecimiento foliar de 45 $\mathrm{cm}$ al día de la cosecha (Gráfica 2 y 3 ), mientras que T2 fue de $38.7 \mathrm{~cm}$ de altura, valores que superan otras investigaciones respecto a los días 8,10 y 12 de cosecha con riego solo de agua la altura es de 13.33 ,
16.60 y $18.66 \mathrm{~cm}$, respectivamente con rendimiento de un kg de maíz a forraje verde hidropónico produce $4.18,4.43$ y $4.78 \mathrm{~kg}$ de eficiencia de conversión (Morales, et al., 2012) mientras que en otro estudio fue de $28.5 \mathrm{~cm}$ para el día 11 (Rivera, et al., 2010), lo anterior se debe a que el desarrollo foliar de la planta es importante elementos como N, P, Ca y Mg (Marschner, 2012) lo cual fue suministrado en la fertilización para los dos tratamientos.

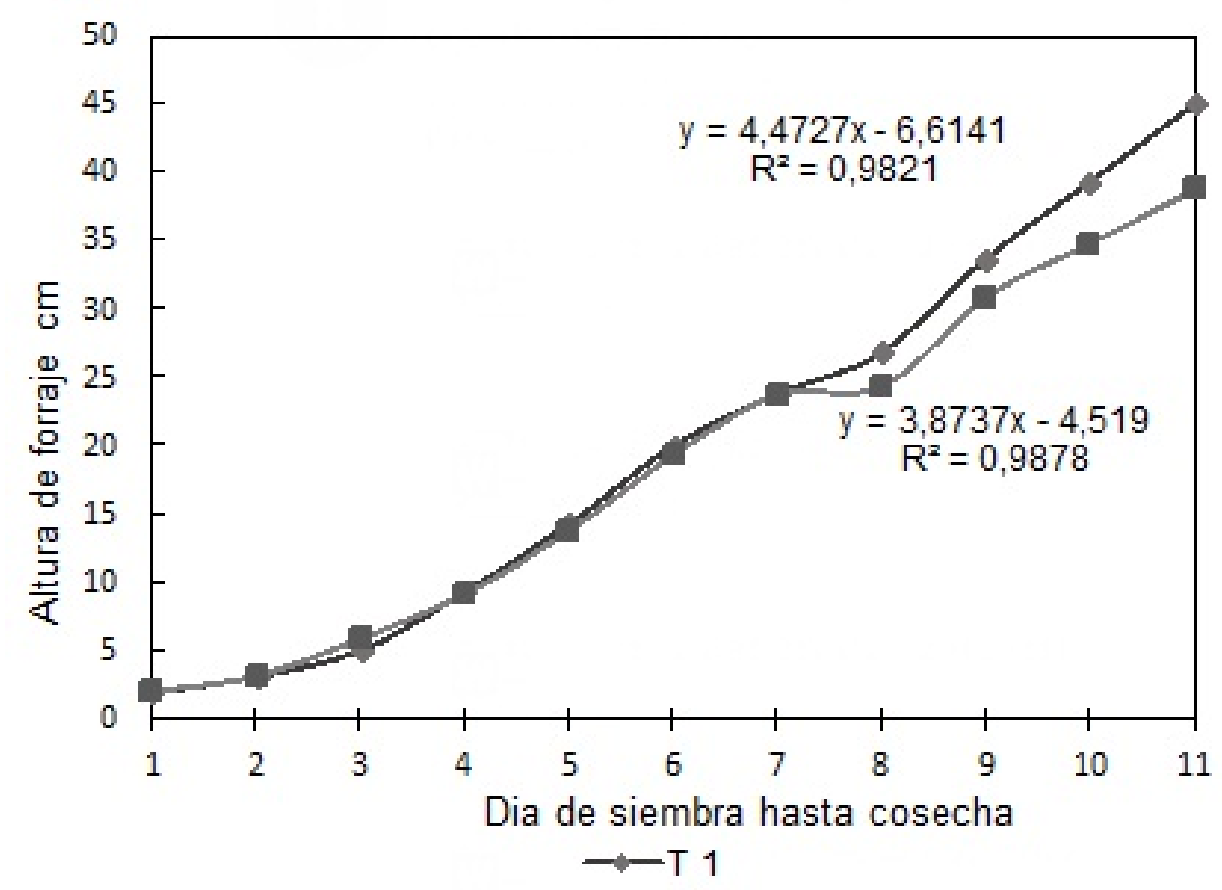

Gráfica 3. Altura de FVH en dos tratamientos

El resultado obtenido del análisis bromatológico en forrajes fue similar (Tabla 3), se evidencia que los tratamientos utilizados para la producción de forraje hidropónico con maíz respecto a la calidad nutricional son adecuados. En el presente estudio los dos tratamientos obtuvieron valores superiores al $7 \%$ de proteína cruda, teniendo en cuenta que la proteína proporciona aminoácidos requeridos para el mantenimiento de las funciones vitales como reproducción, crecimiento y lactancia, de acuerdo con el programa de alimentación bovina los pastos contienen menos del $7 \%$ de proteína que conlleva disminuir el consumo en los animales, por tanto, se estima que cada vez que el contenido de proteína se disminuye en un $1 \%$ por debajo del $7 \%$, el animal baja un 10\% su consumo (Fedegan, 2011), a su vez, Fudge y Fraps (1944) clasifica el contenido de proteína superior al $16.5 \%$ como forraje de excelente calidad ubicando al tratamiento T $1(19.9 \%)$ en esta categoría, mientras que el tratamiento T2 presento un forraje de regular calidad entre $7.5 \%$ y $11.9 \%$. 
Tabla 3. Contenido nutricional de forraje hidropónico de maíz en dos tratamientos NULAB. S.A.S

\begin{tabular}{|c|c|c|c|c|c|c|c|c|c|}
\hline \multicolumn{10}{|c|}{ Análisis bromatológico } \\
\hline Tratamiento & $\begin{array}{c}\text { Caloría } \\
\text { Kcal }\end{array}$ & $\begin{array}{c}\text { Carbohidratos } \\
\%\end{array}$ & $\begin{array}{c}\text { Ceniza } \\
\%\end{array}$ & $\begin{array}{l}\text { Fibra } \\
\text { cruda }\end{array}$ & $\begin{array}{l}\text { Grasa } \\
\text { cruda }\end{array}$ & $\begin{array}{c}\text { Humedad } \\
\%\end{array}$ & $\begin{array}{c}\text { Proteína } \\
\text { Cruda } \\
\text { BS \% }\end{array}$ & $\begin{array}{c}\text { Proteína } \\
\text { Cruda } \\
\text { BH \% }\end{array}$ & $\begin{array}{c}\text { Solidos } \\
\text { Totales } \\
\%\end{array}$ \\
\hline T1 & 43.99 & 6.91 & 1.19 & 2.41 & 0.59 & 86.14 & 19.91 & 2.76 & 13.9 \\
\hline T2 & 75.05 & 14.52 & 0.87 & 3.13 & 0.77 & 78.2 & 11.5 & 2.51 & 21.8 \\
\hline
\end{tabular}

En el parámetro calorías se identificó una diferencia ya que para el tratamiento T1 se obtuvo 43.99 Kcal y T2 de 75.05 Kcal, lo cual nos indica que el contenido de energía es mayor en T2 y por tanto se debe brindar mayor cantidad de alimento para que el animal tenga una dieta balanceada. Por otra parte, el agua no es considerada como un nutriente, pero es vital en el buen funcionamiento de todas las actividades en el cuerpo del bovino, siendo una fuente principal de agua presente en pastos o forrajes, Según Ramírez (2011) el porcentaje de humedad en forrajes apropiado es del $65 \%$ dato superado por los dos tratamientos evaluados siendo un suplemento fresco que otorga $8.1 \%$ para $\mathrm{T} 1$ mientras que el T2 del $78.2 \%$. El porcentaje obtenido a los 11 días de cosecha en fibra cruda del forraje verde hidropónico de maíz fue de $2.41 \%$ (T1) y $3.13 \%$ (T2), estos valores se encuentran por debajo de 8.87 y $9.03 \%$ de fibra cruda obtenido a los 10 días de cosecha con solución nutritiva y sin solución reportado por la FAO (2001). A medida que el forraje madura, aumenta el contenido de fibra y disminuye su contenido de proteína y azúcares, a mayor fibra, menor consumo voluntario del alimento (López, et al., 2009).

\section{Conclusiones}

Los dos tratamientos (Biofertilizante liquido microbiano y solución nutritiva $\mathrm{AB}$ ) presentaron un excelente contenido nutricional para suplementar al ganado bovino con diferencias representativas en las variables evaluadas para T1. Por tanto, se recomienda la implementación de sistemas de fertilización química en producción de forraje verde hidropónico, ya que incrementa el rendimiento por metro cuadrado.

\section{Referencias bibliográficas}

Baixauli, C., Aguilar J., 2002. Cultivo sin suelo de hortalizas: Aspectos Prácticos y experiencias. Valencia, España: Generalitat Valenciana.

Ceballos, C., García, P., 1992. Cultivos hidropónicos. En Howard M. Resh.( Ed), Nuevas técnicas de producción, (pp. 176-178), Madrid, España: Mundi-prensa.

Fedegan., 2011. Federación Nacional de Ganaderos. Programa de alimentación bovina: el ganado paga, pero bien alimentado. Consultado el 15 de febrero de 2019. http://www.fedegan.org.co

Fudge, J., Fraps, G.,1944. The chemical compositions of forage grasses from de Gulf coast praerie as related to soil and to requeriments for range cattle. Texas agricultural experiment station. 644. $39 \mathrm{p}$

Guzmán, G., Pardo, O., Mojica, J., 2003. Ensilaje de cultivos forrajeros para la alimentación de bovinos en el Piedemonte Llanero. Corpoica, 33 (1), 20-30.

Hernández, V., 2016. 7 pasos para la producción de forraje verde hidropónico. Contextoganadero. Consultado el 15 de febrero de 2019. http://www.contextoganadero.com

ICA., 2016. Instituto Colombiano Agropecuario. Censo pecuario nacional: población bovina por municipio y por departamento 2016. Consultado el 15 de febrero de 2019. Recuperado de https://www.ica.gov.co 
López, R., Murillo, B., Rodríguez, G., 2009. El forraje verde hidropónico, una alternativa de producción de alimento para el ganado en zonas áridas. Interciencia, 34 (2). 121-126. Recuperado de http://www.redalyc.org. https://doi.org/10.5377/ ribcc.v5i9.7947

Marschner, H., $2012 . \quad$ Marschner's Mineral Nutrition of Higher Plants. DOI: 10.1017/S001447971100130X

Morales, R., Gómez, A., Juárez L., Loya, O., 2012. Forraje verde hidropónico de maíz amarillo (zea maíz l.) con diferente concentración de solución nutritiva. Abanico Veterinario. 2 (3), 20-28. Consultado el 15 de febrero de 2019. https://abanicoacademico.mx/revistasabanico/. https://doi.org/10.22267/rcia.153201.26

Müller, L. Manfron, P. Santos, O. Medeiros, S. Haut, V. Dourado-Bandeira, A., 2005. Produção e composição bromatológica da forragem hidropônica de milho, Zea mays L., com diferentes densidades de semeadura e datas de colheita. Zootecnia Trop, 23(2), 105-119. Consultado el 15 de febrero de 2019 . https://biblat.unam.mx. https://doi.org/10.1590/s0103-84782006000400008

Organización de las Naciones Unidas para la Agricultura y la Alimentación (FAO). (2001). Manual Técnico: Forraje verde hidropónico. Consultado el 15 de febrero de 2019. http://www.fao.org. https://doi.org/10.18356/a48b842b-es

Pant, A. P., Radovich, T. J., Hue, N. V., Talcott, S. T. and Krenek, K. A. (2009), Vermicompost extracts influence growth, mineral nutrients, phytonutrients and antioxidant activity in pak choi (Brassica rapa cv. Bonsai, Chinensis group) grown under vermicompost and chemical fertiliser. J. Sci. Food Agric.,
89 (14): 2383-2392. DOI:10.1002/jsfa.3732. https://doi.org/10.1002/jsfa.3732

Pasuy, P., Cifuentes,L., Benavides,O., y Viveros, M., (1989). Respuesta del maíz a cuatro concentraciones de una solución nutritiva en condiciones hidropónica y de invernadero en el ciab Pasto. Ciencias Agrícolas 11(8), 56-63. Consultado el 15 de febrero de 2019. http://revistas.udenar.edu.co. https://doi.org/10.15517/rac.v40i2.27360

Ramírez C., Soto, F. 2017. Efecto de la nutrición mineral sobre la producción de forraje verde hidropónico de maíz. Agronomía Costarricense, 41(2). doi:http://dx.doi.org/10.15517/rac.v41i2.313 01

Ramírez, H., 2011. Consejos prácticos ¿De qué hablan cuando dicen Materia Seca?. Engormix. Consultado el 15 de febrero de 2019. https://www.engormix.com

Rivera, A., Moronta, M., González, M., González, D., Perdomo, D., García, D., Hernández, G., 2010, Producción de forraje verde hidropónico de maíz (Zea mays L.) en condiciones de iluminación deficiente. Zootecnia Trop. 28(1): 33-41. https://doi.org/10.22267/rcia.153201.26

Theunissen, J., Ndakidemi, PA., Laubscher, CP., 2010. Potential of vermicomposta produced from waste on the growth and nutrient status in vegetable production. J. Phys. Sci. 5 (19), 64-73.

Vargas, C., 2008. Comparación productiva de forraje verde hidropónico de maíz, arroz y sorgo negro forrajero. Agronomía Mesoamericana, 19 (2), 233-240. Consultado el 15 de febrero de 2019 . https://revistas.ucr.ac.cr. https://doi.org/10.15517/am.v19i2.5005

La Revista Ingeniería y Región cuenta con la Licencia Creative Commons Atribución (BY), No Comercial (NC) y Compartir Igual (SA) 